\title{
MODELLING WAVE TRANSFORMATION ACROSS A FRINGING REEF USING SWASH
}

\author{
Marcel Zijlema ${ }^{1}$
}

\begin{abstract}
This paper presents the application of the open source non-hydrostatic wave-flow model SWASH to wave propagation over a fringing reef, and the results are discussed and compared with observations obtained from a laboratory experiment subjected to various incident wave conditions. This study focus not only on wave breaking, bottom friction, and wave-induced setup and runup, but also on the generation and propagation of infragravity waves beyond the reef crest. Present simulations demonstrate the overall predictive capabilities of the model for a typical coral reef with steep slopes and extended reef flats.
\end{abstract}

Keywords: fringing reefs; wave breaking; bottom friction; infragravity waves; runup; SWASH

\section{INTRODUCTION}

Fringing coral reefs are the most common reef type and are located nearshore in the tropical regions. They are characterized by a steep slope with a transition at the reef crest to a shallow flat attached to the shoreline. Intense wave breaking typically occurs around the reef crest and protects the coastline from severe wave conditions, but increases the water level due to wave setup. Over the flat, the bottom roughness associated with the reef formation and corals can be very dominated and may dissipate as much energy as wave breaking. Triad interactions often cause dramatic alteration of the spectral shape across the reef. Energy is being transferred from the spectral peak to the super harmonics during shoaling and then toward the infragravity band in the surf zone.

The interaction between waves and fringing reef structures is complex and is an area of ongoing research. Many of the experimental and numerical studies that have been conducted concerning coral reefs have focused on understanding the processes and interactions of wave and current hydrodynamics in the presence of a reef. See Monismith (2007) for a review. Yet a number of different numerical wave models have been utilized to describe specific processes related to reefs, e.g. wave breaking, nonlinear energy transfer and setup. These models are typically semi empirical with the required parameterization for nonlinear transfer and wave energy dissipation caused by wave breaking and bottom friction. For instance, Massel and Gourlay (2000) have employed an extended linear mild-slope model for steep slopes to predict wave breaking and setup on coral reefs of various shapes. This model is calibrated by optimizing dissipation parameters based on minimizing least square error. Reasonable agreement has been reported in Sheremet et al. (2011) in which they have applied two nonlinear spectral models to study transformation of wave spectrum over fringing reefs. An inversed modelling approach has been used to calibrate a number of wave breaking parameters. Recently, Filipot and Cheung (2012) have assessed the performance of the $3^{\text {rd }}$ generation spectral wave model SWAN for application in fringing reef environments. They have examined a number of embedded parameterizations of wave breaking, bottom roughness and nonlinear energy transfer (to the super harmonics), and subsequently have recalibrated these parameterizations to improve the overall model performance. Skotner and Apelt (1999) and Nwogu and Demirbilek (2010) investigated the ability of a Boussinesq-type wave model upon a Guam reef profile. The rate of wave energy dissipation has been determined by tuning several wave breaking parameters (e.g. surface roller and turbulent length scale) and bottom friction coefficient. Recently, a shock-capturing Boussinesq-type wave model enabling to account for more realistic wave breaking has been applied to simulate nearshore wave processes over fringing reefs with different shape profiles (Roeber and Cheung 2012).

Limited research has been conducted into the interaction of low frequency waves with coral reefs. This is may be due to lack of suitable data and some limitations imposed in many numerical models. Nevertheless, the importance of infragravity waves has been recognised for reefs. For instance, the observation by Nwogu and Demirbilek (2010) that excitation of infragravity waves is possible when a natural frequency is present demonstrated the importance of understanding the interaction of low frequency waves in reef environments. Using laboratory tests of Demirbilek et al. (2007) and a Boussinesq-type wave model, they found that low frequency waves could be resonantly amplified at the shoreline with the first reef oscillation mode having a wave length approximately equal to four times the

\footnotetext{
${ }^{1}$ Environmental Fluid Mechanics Section, Faculty of Civil Engineering and Geosciences, Delft University of Technology, P.O. Box 5048, 2600 GA Delft, The Netherlands
} 
width of the reef flat. Furthermore, it was found that wave motions over the reef flat and at the shoreline were dominated by oscillations at high wave periods as most of the incident wave energy is dissipated within a few wave lengths of the reef face. The low frequency wave energy was found to be minimum at the reef crest and then increased as the wave propagated shoreward over the reef flat. The wave setup was shown to increase in a similar way.

A comparison with the laboratory data of Demirbilek et al. (2007) for wave transformation over a shallow fringing reef, and in particular low frequency motion across a reef flat, allows an assessment of the non-hydrostatic wave-flow SWASH model. This model is an open source tool developed at Delft University of Technology (http://swash.sf.net) and it was demonstrated to be capable to model low frequency waves and the subsequent hydrodynamic processes in the sandy coast environment (see, e.g. Zijlema et al. 2011; Rijnsdorp et al. 2012). In this study, the validity of SWASH in describing wave transformation across fringing reefs and reproducing the interaction between low frequency waves and the reef will be examined. This will identify the capabilities and limitations of SWASH in relation to modelling low frequency wave interaction with reef type structures.

\section{SWASH: A NON-HYDROSTATIC WAVE-FLOW MODEL}

SWASH (an acronym of Simulating WAves till SHore) takes as its starting point the incompressible Navier-Stokes equations or Euler equations for the computation of the surface elevation and currents. In fact, these equations can be regarded as nonlinear shallow water (NLSW) equations including the effect of vertical acceleration. For the present purpose of outlining the principles adopted, the precise form of the governing equations is irrelevant. However, one is refer to Zijlema and Stelling (2005) and Zijlema et al. (2011) for details. Also, details on the imposition of the boundary conditions can be found in Zijlema et al. (2011) and Rijnsdorp et al. (2012).

The numerical framework of SWASH has been extensively presented and discussed in Zijlema and Stelling (2005) and Zijlema et al. (2011). In this section, a brief outline of some numerical procedures relevant to the surf zone applications is given.

SWASH employs an explicit, second order finite difference method for staggered grids. This framework is the most natural and advantageous basis for advanced wave modelling in coastal areas. In addition, a discretized form of the NLSW equations can automatically be shock-capturing if the momentum conservation is retained in the finite difference scheme. The principle of this approach, as well as its underlying rationale are documented in Stelling and Duinmeijer (2003), Zijlema and Stelling (2008) and Zijlema et al. (2011). In the vertical, the computational domain can be divided into a fixed number of terrain-following layers. Space discretization in the vertical direction is carried out in a finite volume fashion. For details, see Zijlema and Stelling (2005).

With respect to time integration of the continuity and horizontal momentum equations, a second order leapfrog scheme known as the Hansen scheme is adopted so that the wave amplitude will not altered (Wesseling 2001). This scheme requires less storage compared to the classical leapfrog scheme and makes the algorithm easy to implement. A MacCormack predictor-corrector technique is employed in order to retain second order accuracy in time for the advection terms in the horizontal momentum equations.

Local mass continuity is enforced by solving a Poisson equation for the pressure correction which steers the non-hydrostatic pressure towards a state at which all mass residuals in the active grid cells become negligible small, reflecting a satisfaction of local mass conservation. Global mass conservation is obtained by solving a depth-averaged continuity equation for the solution of the surface elevation.

In order to resolve the frequency dispersion up to an acceptable level of accuracy using as few layers as possible, a technique as proposed in Stelling and Zijlema (2003) is used that is tailored to coastal wave propagation. It is based on a compact Keller-box difference scheme for the approximation of vertical gradient of the non-hydrostatic pressure. A main advantage of this scheme is to provide a much better representation of the shorter length scales of solution compared to the classical finite difference schemes. This better representation can be attributed to the implicit nature of the box scheme which makes such a scheme more accurate than standard schemes on relatively coarse grids even with the same order of accuracy. As a result, accurate propagation of progressive waves for $k d \leq 3$ ( $k$ is the wave number, $d$ is the still water depth) can be obtained with just two equidistant layers.

Given a limited amount of vertical layers, i.e. 1-3 layers, the adopted momentum-conservative scheme is able to track the actual location of incipient wave breaking accurately, but generally underestimates the subsequent dissipation of wave energy. This is due, principally, to the modest levels of amplitude dispersion and, at the same time, the excessive levels of frequency dispersion up to the 
breakpoint, preventing the wave from transitioning into the characteristic saw-tooth shape, and therefore postponing the onset of dissipation. This defect is rooted in the underestimation of the horizontal velocity near the crest of the breaking wave due to the coarse vertical resolution. A simple approach is to locally impose a hydrostatic pressure distribution under the wave front (Smit et al. 2012). By doing this, the bore-like wave front steepens continuously until it becomes vertical. Subsequently, the broken wave propagates with a correct gradual change of form and resembles a steady bore in a final stage. This leads to a correct amount of energy dissipation on the front face of the breaking wave. This is supported by the successful application of the NLSW equations in the surf zone; e.g. Peregrine (1983). Moreover, intra-phase properties such as asymmetry and skewness are preserved as well. In practice, this very simple treatment of wave breaking does not require any additional calibration.

Finally, a very simple wet-dry approach as treated in Stelling and Duinmeijer (2003) is adopted. This method tracks the motion of the shoreline very accurately without posing numerical instabilities by ensuring non-negative water depths and using the upwind water depths in the momentum flux approximations.

\section{LABORATORY TEST CASES}

Demirbilek et al. (2007) reported a flume experiment on transformation of unidirectional, irregular waves over a fringing reef. The flume, which is $35 \mathrm{~m}$ long, $0.7 \mathrm{~m}$ wide, and $1.6 \mathrm{~m}$ high, features a 1:64 scaled model of a reef typical of the southeast coast of Guam. The corresponding bottom topography is shown in Fig. 1 in which also the locations of nine wave gauges are indicated. The measured surface elevations at gauge no. 4 appeared to be faulty and are therefore not used here. Next, a sensor was installed on the beach to measure runup height. A plunger-type wave maker was located at $15.5 \mathrm{~m}$ from the toe of the reef and generated irregular sea states based on a JONSWAP spectrum with peak enhancement factor 3.3.

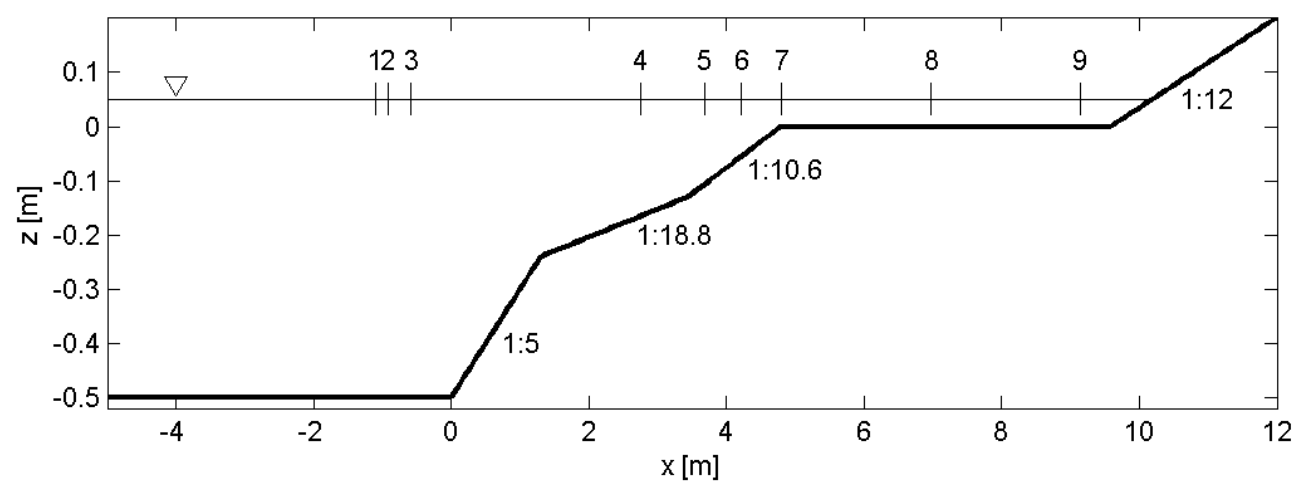

Figure 1. Bottom topography and location of wave gauges of the idealized reef of Demirbilek et al. (2007). This reef includes a composite slope for the reef face, a reef flat, and a beach.

Laboratory tests were conducted in the flume with different combinations of significant wave height $H_{\mathrm{m} 0}$, peak period $T_{\mathrm{p}}$, and still water depth over the reef flat $h_{\mathrm{r}}$. Some of these input conditions were selected for the validation of SWASH. They are listed in Table 1.

\begin{tabular}{|c|c|c|c|c|c|}
\hline \multicolumn{6}{|c|}{ Table 1. Input conditions for the tests of Demirbilek et al. (2007). } \\
\hline Test no. & $H_{\mathrm{m} 0}(\mathrm{~cm})$ & $T_{\mathrm{p}}(\mathrm{s})$ & $h_{\mathrm{r}}(\mathrm{cm})$ & $k_{\mathrm{p}} h_{0}$ & $\xi_{0}$ \\
\hline 20 & 6.1 & 1.25 & 5.1 & 1.55 & 0.54 \\
17 & 7.8 & 1.50 & 5.1 & 1.19 & 0.55 \\
21 & 8.2 & 1.75 & 5.1 & 0.97 & 0.59 \\
18 & 8.5 & 2.00 & 5.1 & 0.82 & 0.63 \\
\hline 46 & 5.9 & 1.25 & 3.1 & 1.51 & 0.56 \\
48 & 7.5 & 1.50 & 3.1 & 1.16 & 0.57 \\
57 & 7.7 & 1.75 & 3.1 & 0.95 & 0.62 \\
58 & 8.5 & 2.00 & 3.1 & 0.80 & 0.64 \\
\hline 27 & 5.5 & 1.25 & 1.6 & 1.48 & 0.59 \\
29 & 7.1 & 1.50 & 1.6 & 1.14 & 0.59 \\
30 & 7.6 & 1.75 & 1.6 & 0.93 & 0.63 \\
31 & 8.5 & 2.00 & 1.6 & 0.78 & 0.65 \\
\hline 36 & 6.8 & 1.50 & 0.0 & 1.11 & 0.61 \\
37 & 7.6 & 1.75 & 0.0 & 0.91 & 0.64 \\
38 & 8.4 & 2.00 & 0.0 & 0.77 & 0.66 \\
\hline
\end{tabular}


This table also indicates the dimensionless depth $k_{\mathrm{p}} h_{0}\left(k_{\mathrm{p}}\right.$ is the wave number associated with the peak period and $h_{0}$ is the water depth at the wave maker), which varies from 0.77 to 1.55 , and the surf similarity parameter $\xi_{0}$ (defined as $\xi_{0}=\tan \beta / \sqrt{H_{\mathrm{m} 0} / L_{\mathrm{p}}}$ with $\tan \beta(=1: 10.6)$ the bottom slope and $L_{\mathrm{p}}$ the wave length associated with the peak period at the wave maker) ranging from 0.54 to 0.66 . Hence, the wave conditions considered are plunging breakers $\left(0.5<\xi_{0}<3.3\right)$.

\section{MODEL SETUP}

Numerical simulations were performed with SWASH in a one-dimensional mode using a grid size of $5 \mathrm{~cm}$, one layer, and an initial time step of $5 \mathrm{~ms}$. The Courant number was 0.5 and the simulation period was $900 \mathrm{~s}$, which was long enough to get a steady-state condition. The time series of measured surface elevation at gauge no. 1 was used to impose the proper boundary conditions for the model. Additionally, reflections of offshore-directed long waves at the incident boundary were minimized. Sponge layers were not employed.

No calibration nor tuning has been carried out in the course of the simulations except for the bottom friction formulation. Contrary to sandy coasts, over reef flats, the bottom roughness may dissipate as much energy as wave breaking. Bottom friction is also important for low frequency motions on reef flats and during wave runup. The bottom stress $\tau_{\mathrm{b}}$ is added to the momentum equations to mimic energy dissipation in the bottom boundary layer, and is based upon a quadratic drag law

$$
\tau_{b}=c_{f} \frac{U|U|}{h}
$$

where $U$ is the depth averaged current, $h$ is the water depth, and $c_{\mathrm{f}}$ is a dimensionless friction coefficient. In the present study we calculate the friction coefficient from the Manning's roughness coefficient $n$, as follows,

$$
c_{f}=\frac{n^{2} g}{\sqrt[3]{h}}
$$

Since the Manning's coefficient $n$ is not known beforehand, we obtained this coefficient after calibration. For this, one case was arbitrarily chosen, namely test no. $48\left(H_{\mathrm{m} 0}=7.5 \mathrm{~cm}, T_{\mathrm{p}}=1.5 \mathrm{~s}\right.$, and $h_{\mathrm{r}}=3.1 \mathrm{~cm}$; cf. Table 1). The best match between model results and observations was obtained with a Manning coefficient of $n=0.01 \mathrm{~s} / \mathrm{m}^{1 / 3}$. This relatively low value can be expected as the reef surface was built using a plastic material (Demirbilek et al. 2007). This value was adopted for the simulation of the other test cases.

\section{RESULTS AND DISCUSSION}

Four tests were selected for presentation of comparison between model and data, as indicated in red in Table 1. The selected cases cover a wide range of both the dimensionless depth and the surf similarity parameter. Also, cases 18 and 38 are more or less comparable, except that the reef flat in test no. 38 is initially dry. This is particularly useful for the verification of the wetting and drying algorithm of SWASH.

The computed and measured wave heights across the flume are compared in Fig. 2 for the selected cases. SWASH captures the overall wave height variations very well. Additionally, the wave height variations indicate that the model reproduces the rapid dissipation of energy during wave breaking. After breaking, waves reform as bores and propagate across the reef flat to the beach. Undular, turbulent bores were observed over the flat (Demirbilek et al. 2007).

Fig. 3 shows the computed and observed wave setup induced by breaking waves. Clearly, small negative setup in the vicinity of breaking and high rising water level on the reef flat is predicted by the model correctly, though the setup on the flat is in some cases slightly overestimated. The decrease in the measured mean water level offshore of the reef was due to the use of a closed laboratory flume with no recirculation of the water pumped onto the reef (Nwogu and Demirbilek 2010). 

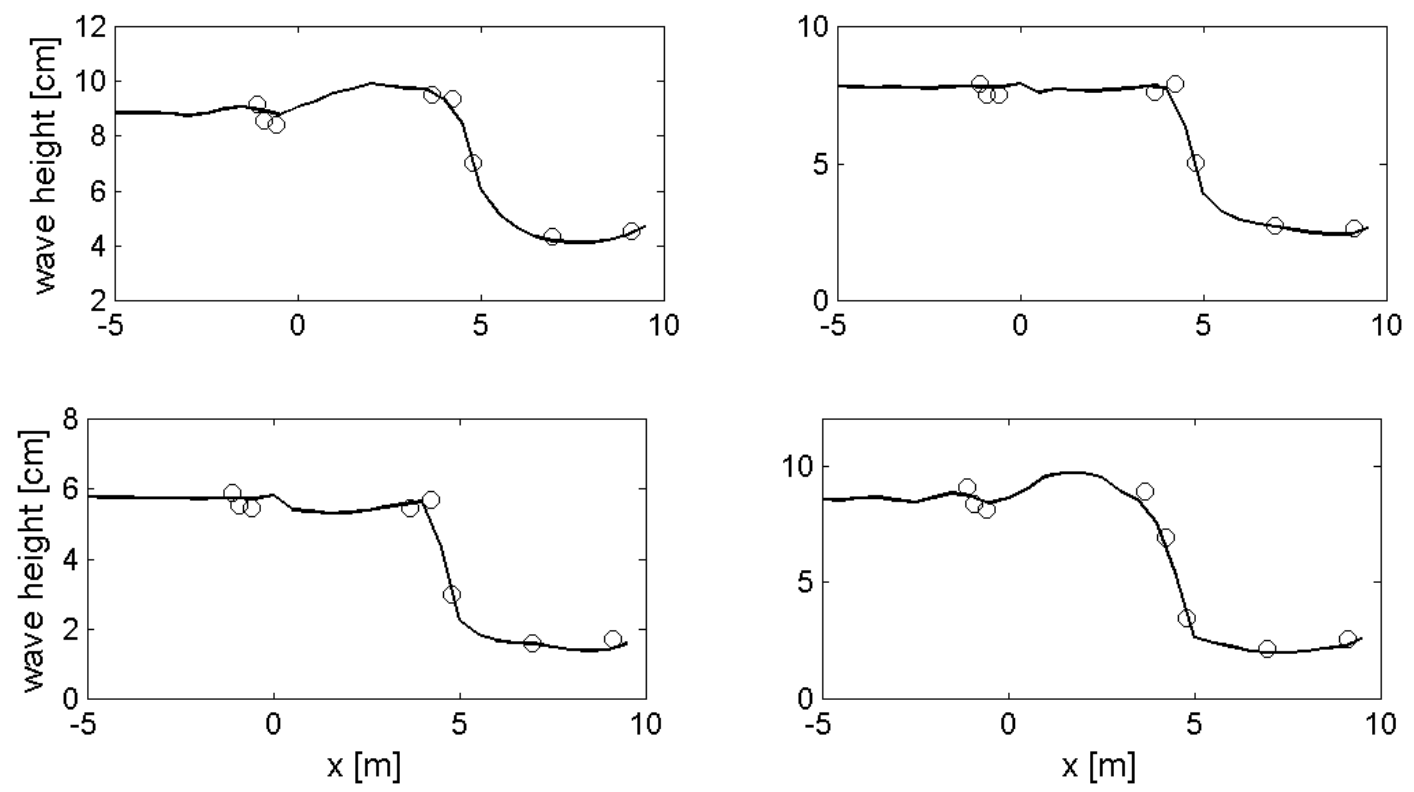

Figure 2. Computed and measured significant wave heights for cases no. 18 (upper-left panel), no. 48 (upper-right panel), no. 27 (lower-left panel), and no. 38 (lower-right panel) along the reef flume. Circles: experimental data; solid line: SWASH.
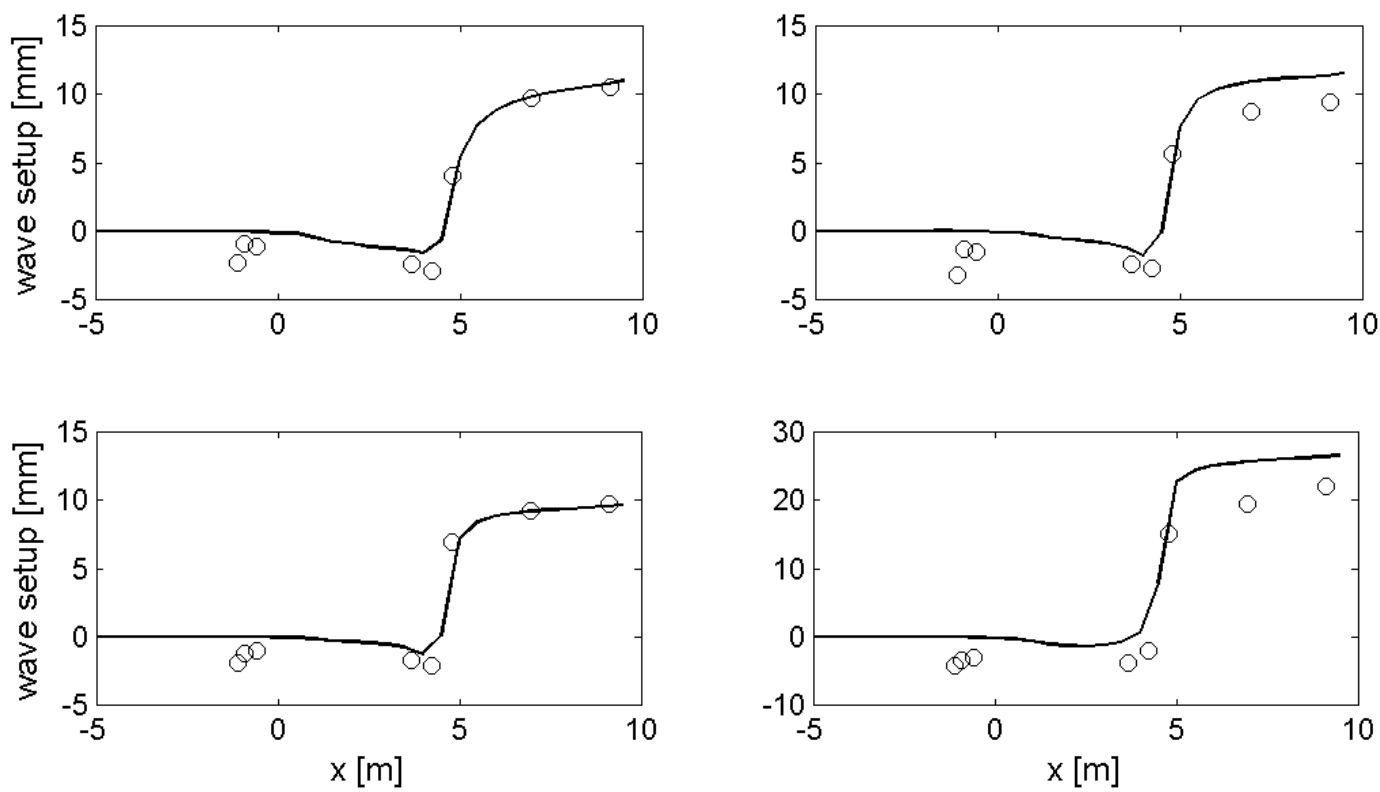

Figure 3. Computed and measured wave setup for cases no. 18 (upper-left panel), no. 48 (upper-right panel), no. 27 (lower-left panel), and no. 38 (lower-right panel) along the reef flume. Circles: experimental data; solid line: SWASH.

Fig. 4a depicts the computed and measured spectra at gauge locations 3 (offshore), 6 (reef-face), 8 (mid-reef flat) and 9 (end of the reef) for test no. 18. Spectra were obtained by Fourier transformation of time series from $100 \mathrm{~s}$ to $900 \mathrm{~s}$. In the present study we shall focus on the spectral energy evolution in the infragravity band over the reef flat. Therefore, the spectra are plotted on a semi-log graph. The spectral evolution agree well with observations, except for the higher harmonics. The underestimation of the computed energy at relative high frequencies was due to the numerical dissipation related to the coarseness of the spatial resolution in the model. Indeed, refining the grid (from 5 to $1 \mathrm{~cm}$ ) improved the spectral shape at high frequencies (see upper-left panel of Fig. 4a). In addition, some of the infragravity energy are overestimated at the offshore location. This was due to the boundary forcing 
based on linear wave theory, which generated spurious free waves at the same frequency as the bound harmonics. Again, we focus on the low-frequency motions propagating over the reef flat.
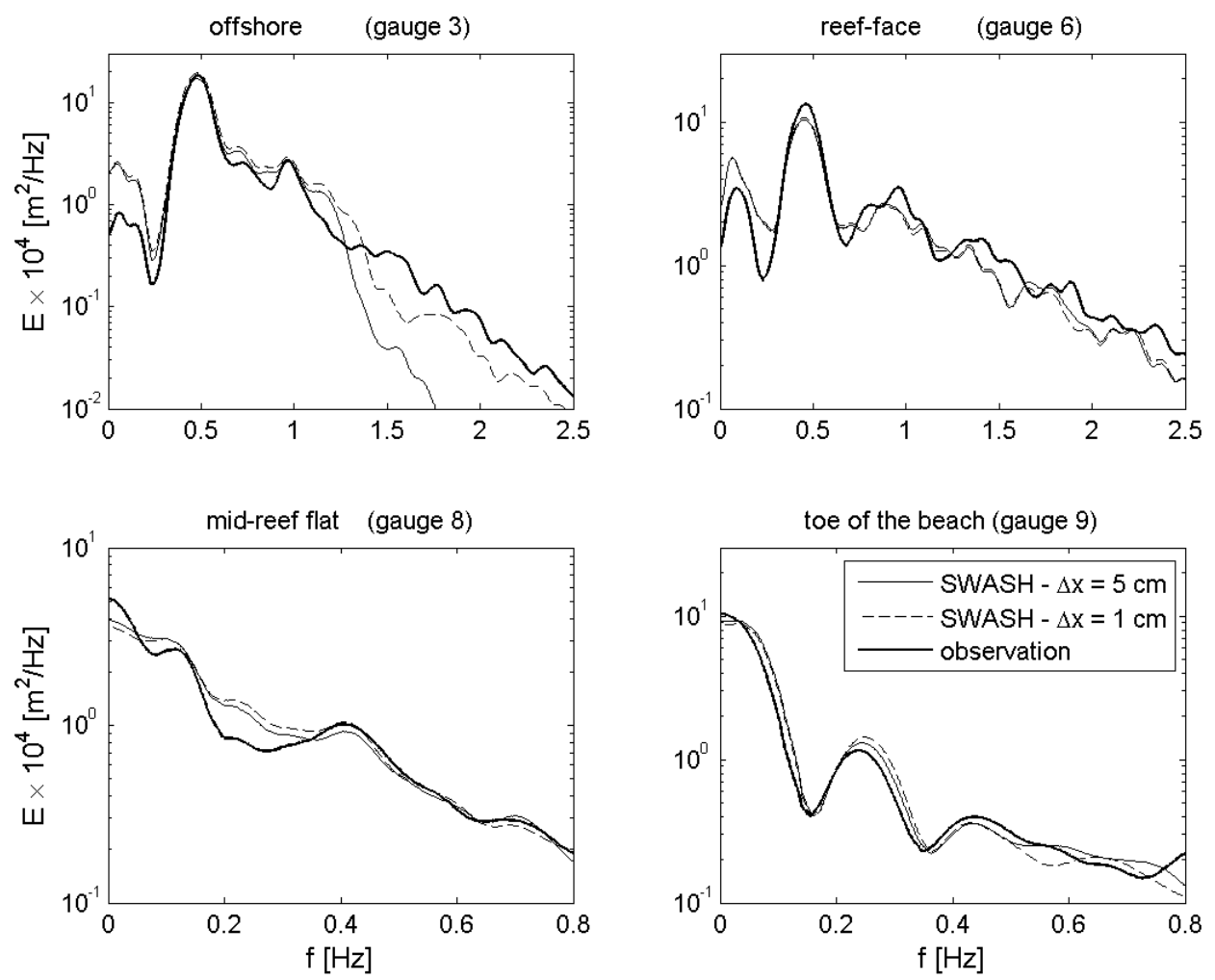

Figure 4a. Comparison of measured and computed wave spectra at selected locations for test no. 18. Thick line: measured data; thin and dashed lines: SWASH.

Clearly, as the waves propagated, energy is transferred from the spectral peak to both bound sub and super harmonics. In particular, the spectra at the reef flat (locations 8 and 9) are dominated by infragravity motions. In addition, most of the energy around the peak of incident waves is dissipated by wave breaking.

A significant part of the energy is shifted toward the infragravity band. This is depicted in Fig. 4b. Generally, the infragravity wave energy is lowest at the reef edge and increases shoreward over the reef flat. Furthermore, the most dominated low-frequency motions at the reef flat (gauges 8 and 9) occur at $f \approx 0.03 \mathrm{~Hz}(\mathrm{~T} \approx 33 \mathrm{~s})$. This corresponds more or less to the first reef mode. A fringing reef structure may be consider as an open basin having a natural resonance frequency equivalent to a wave length of four times the width of the reef flat:

$$
T_{\mathrm{n}}=\frac{4 l_{\mathrm{r}}}{(2 n-1) \sqrt{g h_{\mathrm{r}}}}, \quad n=1,2,3, \ldots
$$

with $T_{\mathrm{n}}$ the standing wave period associated with mode $n$ for a reef of width $l_{\mathrm{r}}(=4.8 \mathrm{~m})$. The first mode $(n=1)$ with $T_{1}$ of $27 \mathrm{~s}$ has a node at the reef edge and an anti-node at the shoreline. Thus, the dominated low-frequency motions trapped over the reef flat and resonantly amplified at the shoreline compared to that of the offshore. These same findings have previously been presented by Nwogu and Demirbilek (2010) using their Boussinesq-type wave model. 

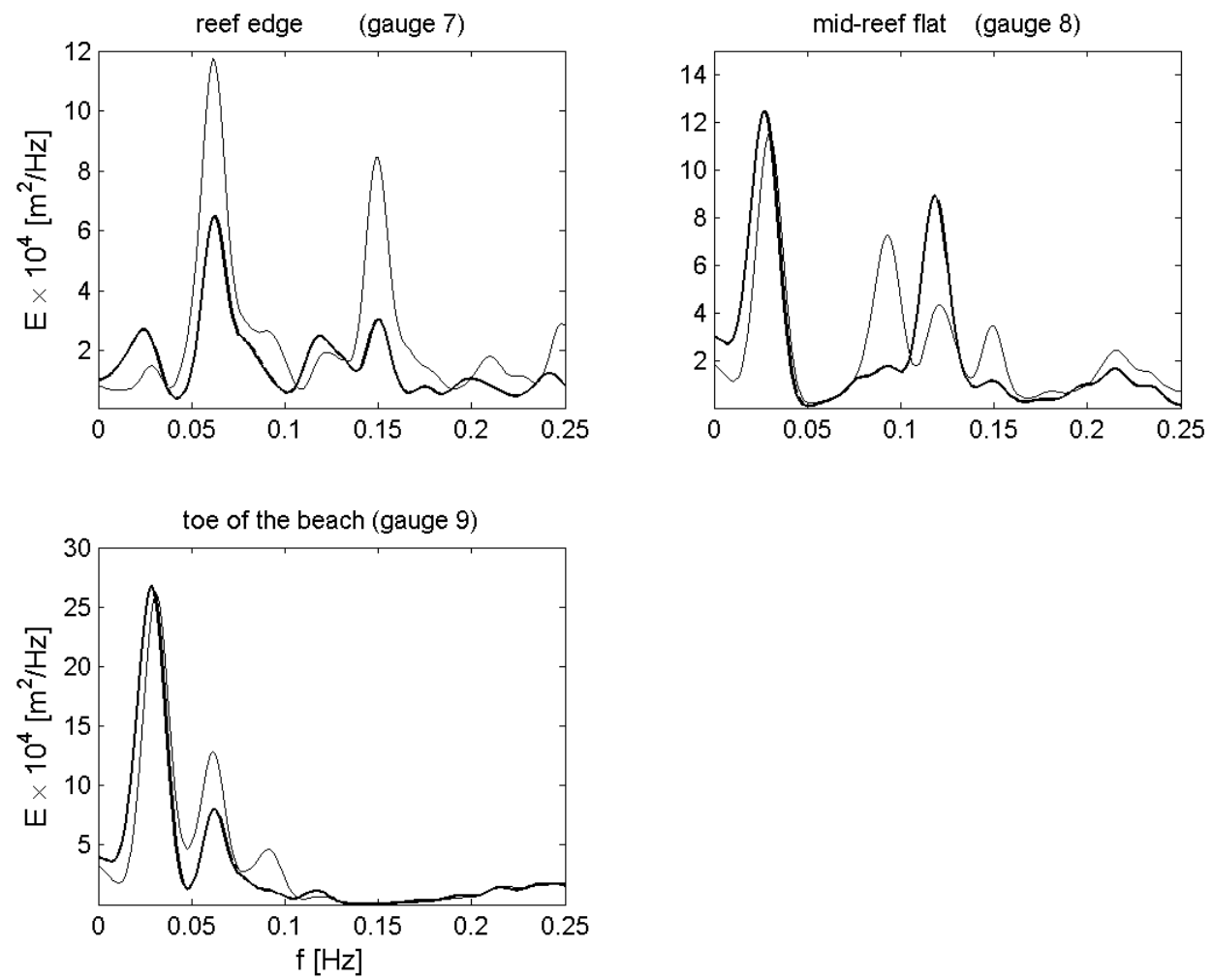

Figure 4b. Comparison of measured and computed wave spectra in the infragravity band at selected locations for test no. 18. Thick line: measured data; thin line: SWASH.

Figs. 5 and 6 compare the computed and recorded time series of the surface elevation for cases no. 18 and 38 at the same locations where the spectra have been composed. These cases have more or less the same wave conditions. The main difference is the water depth over the reef flat (see Table 1). These plots provide further evidence that SWASH is able to reproduce the overall trends in the nonlinear energy transfer over the fringing reef. For instance, the model reproduces the nonlinear steepening and asymmetry after the waves have broken. Moreover, the model is able to describe both the amplitude and phase of the infragravity oscillations over the reef flat. Obviously, the low-frequency motions are stronger for reefs exposing at low water level compared to that of high water level. 

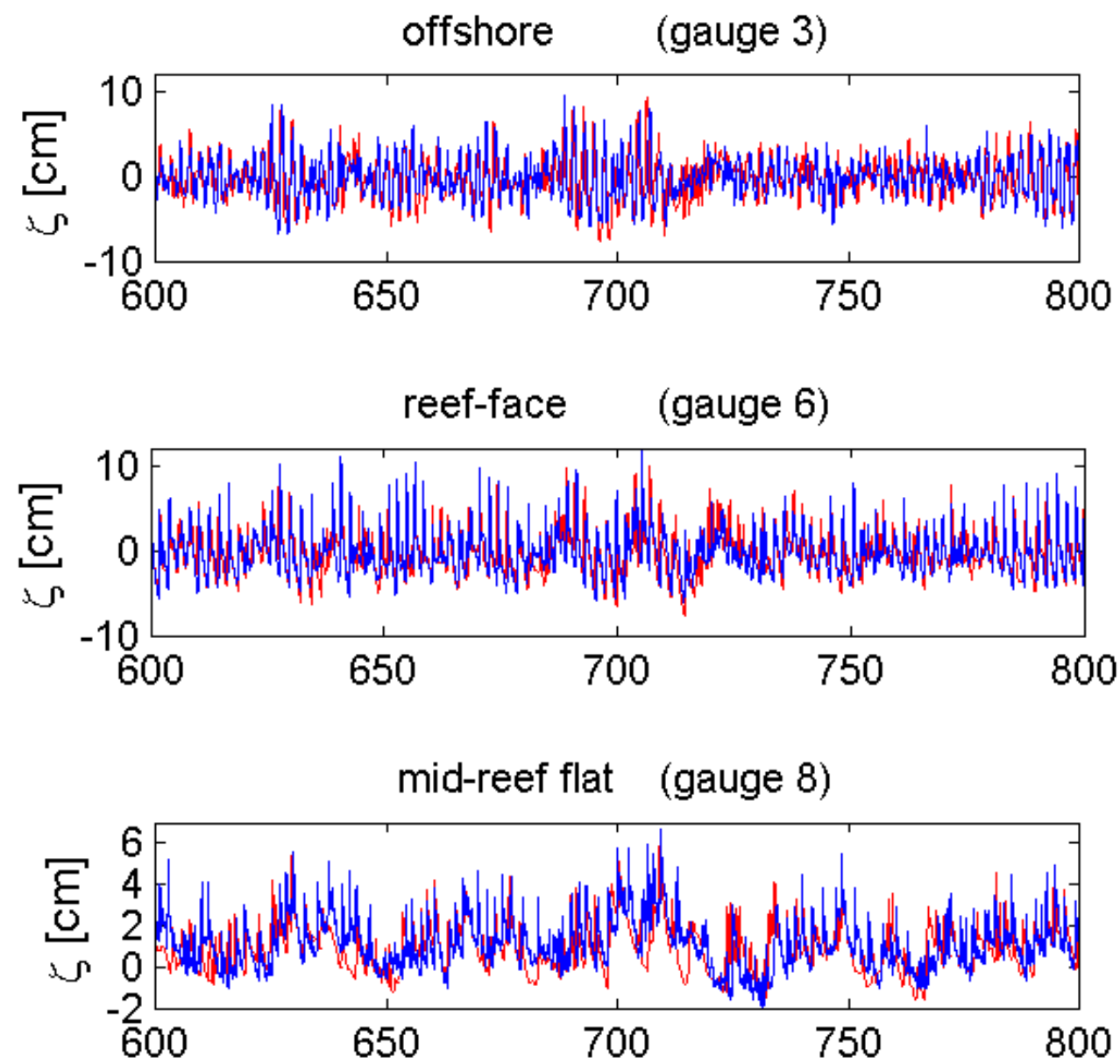

toe of the beach (gauge 9 )

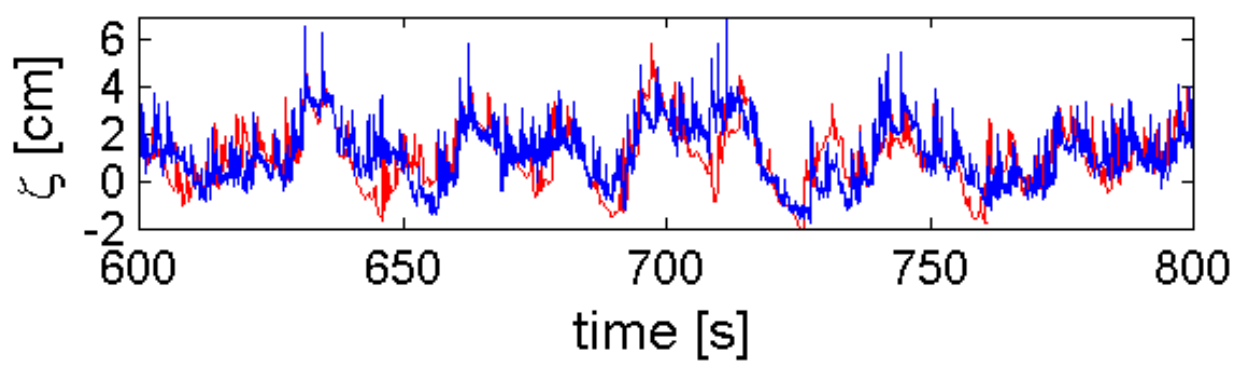

Figure 5. Comparison of recorded (blue) and predicted (red) time histories of surface elevation at selected gauges across the reef topography for test no. 18. 

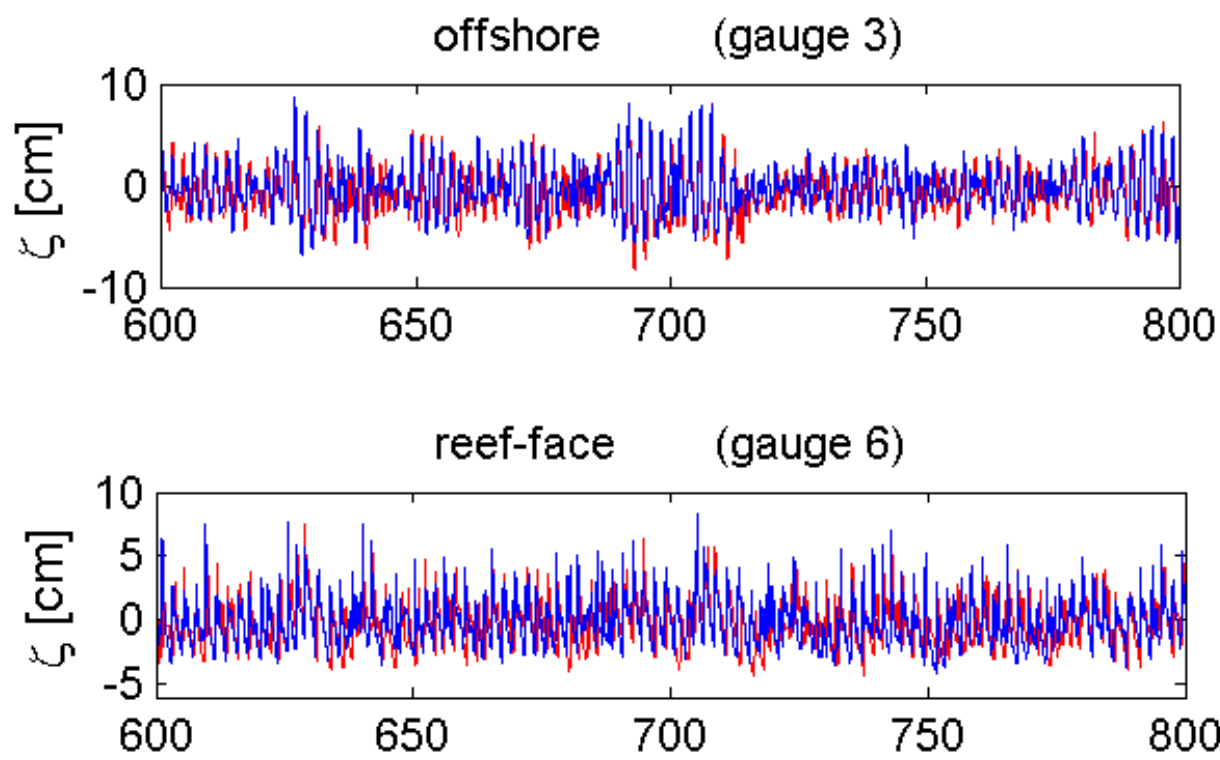

mid-reef flat (gauge 8)

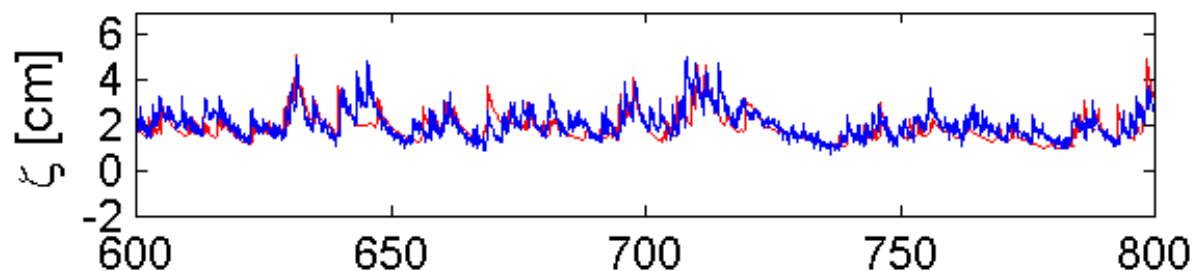

toe of the beach (gauge 9)

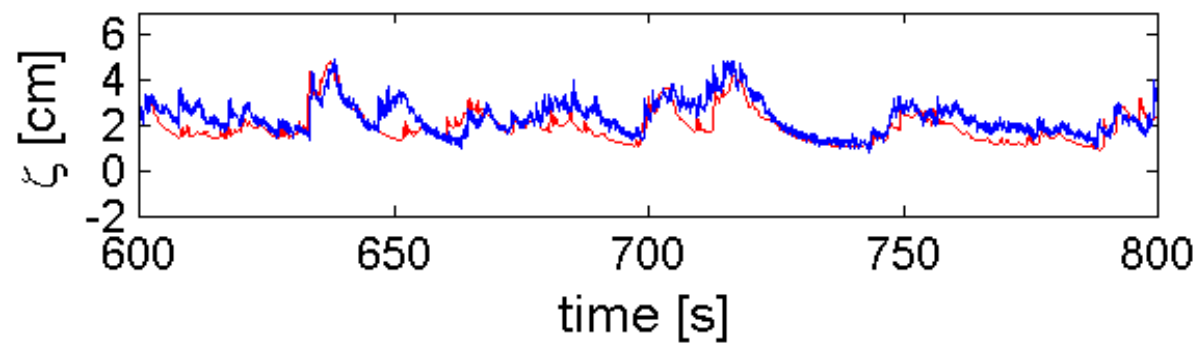

Figure 6. Comparison of recorded (blue) and predicted (red) time histories of surface elevation at selected gauges across the reef topography for test no. 38 . 
The predictive skill of the SWASH model was determined by measuring the bias and the scatter index SI. These are given by

$$
\text { bias }=\frac{1}{N} \sum_{i=1}^{N}\left(\varphi_{\mathrm{comp}}^{i}-\varphi_{\mathrm{obs}}^{i}\right)
$$

and

$$
\mathrm{SI}=\frac{\sqrt{\frac{1}{N} \sum_{i=1}^{N}\left(\varphi_{\mathrm{comp}}^{i}-\varphi_{\mathrm{obs}}^{i}\right)^{2}}}{\frac{1}{N} \sum_{i=1}^{N} \varphi_{\mathrm{obs}}^{i}}
$$

where $\varphi_{\text {obs }}$ is the observed wave parameter, $\varphi_{\text {comp }}$ is the corresponding value computed by SWASH and $N$ is the total number of data points in a given data set. These statistical measures were determined for the significant wave height $H_{\mathrm{m} 0}$ and the wave-induced setup, and were computed for all considered test cases at each location. Note that only the wave setup at the reef flat (gauges 7, 8 and 9) is considered, since there is almost no setup at the reef face and offshore of the reef. Also note that data from gauge 4 are not included as this gauge appeared to be inaccurate (Demirbilek et al. 2007). The results are

\begin{tabular}{|c|c|c|c|c|}
\hline gauge & $\begin{array}{c}\text { bias } H_{\mathrm{m} 0} \\
(\mathrm{~mm})\end{array}$ & $\mathrm{SI} H_{\mathrm{m} 0}$ & $\begin{array}{l}\text { bias setup } \\
(\mathrm{mm})\end{array}$ & SI setup \\
\hline 1 & -1.73 & 0.024 & - & - \\
\hline 2 & 3.26 & 0.044 & - & - \\
\hline 3 & 3.04 & 0.042 & - & - \\
\hline 5 & 1.07 & 0.039 & - & - \\
\hline 6 & -3.80 & 0.057 & - & - \\
\hline 7 & -4.27 & 0.098 & -3.50 & 0.476 \\
\hline 8 & -0.97 & 0.056 & -1.71 & 0.165 \\
\hline 9 & -1.85 & 0.076 & -2.26 & 0.193 \\
\hline
\end{tabular}
presented in Table 2.

Overall, the comparison between computed and observed wave heights is good at all locations for all considered wave conditions. In fact, relatively small hindcast bias is computed for the wave height (less than $5 \mathrm{~mm}$ which is small compared to a typical wave height of 5 to $10 \mathrm{~cm}$ ). Considering the scatter index the highest value is $9.8 \%$ found at gauge 7 (reef edge). Obviously, the lowest error of $2.4 \%$ is found offshore. On average, the rms-error is $5.5 \%$, which is highly acceptable. Clearly, the computed wave setups are systematically lower than the measured values. On the other hand, the highest error is $47.6 \%$ at gauge 7 . This is probably related to the wave breaking mechanism handled in the model. Nevertheless, computed and observed wave setups on the reef top at locations 8 and 9 are in good agreement as the scatter index is $16.5 \%$ and $19.3 \%$, respectively, which is acceptable.

A distinguished runup characteristic is the maximum runup height, which is an important factor in determining flooding and erosion of reef-protected coasts. Measured and predicted maximum runup heights for the 15 test conditions considered are compared in Fig. 7 showing that they are in good agreement (rms-error of $15 \%$ and a very mild bias of $5 \mathrm{~mm}$ ). 


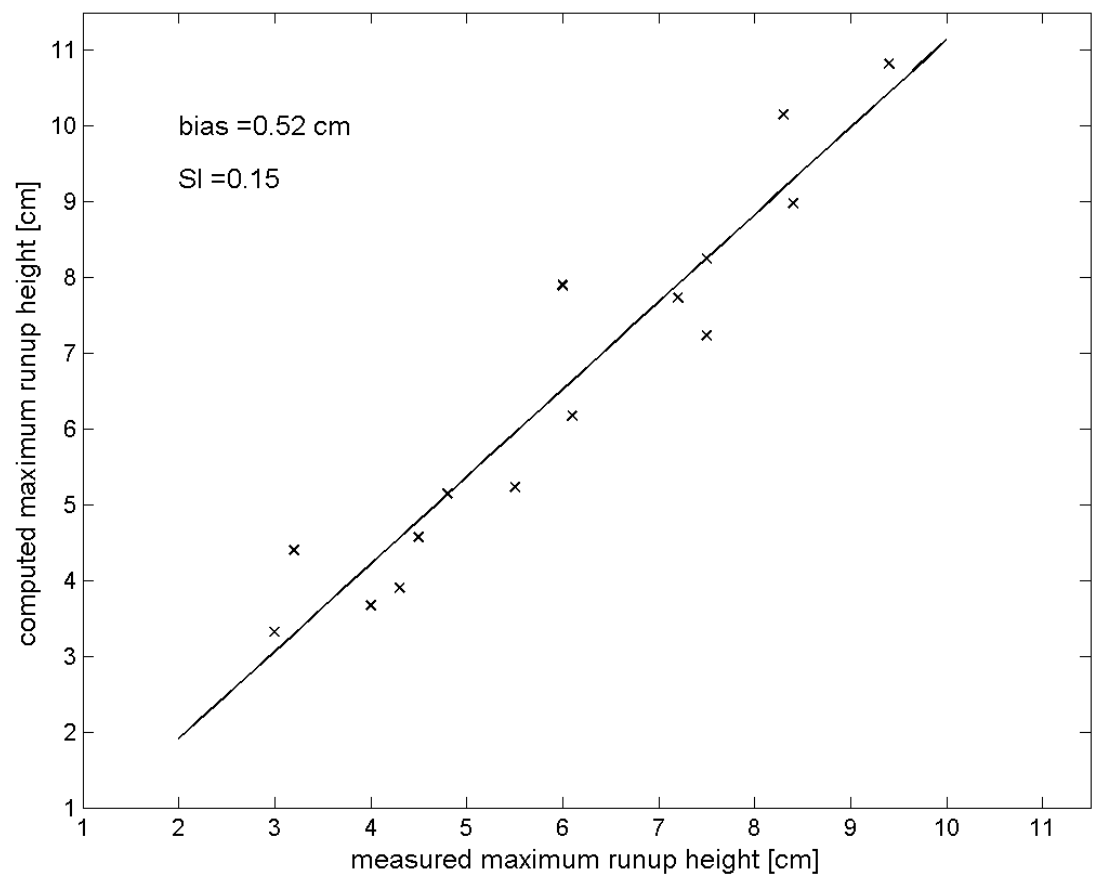

Figure 7. Scatter plot for maximum runup height at runup gauge on the beach.

\section{CONCLUSIONS}

A validation study of SWASH has been undertaken for wave transformation over an idealized fringing reef with different incident wave conditions and still water depths over the reef flat. It was found that SWASH, in general, reproduced the wave height, setup and runup measured in the experiments, and described the associated processes of energy dissipation and nonlinear energy transfer considerably well. For instance, SWASH was able to capture the sharp energy decay of the wave height associated with the energetic breaking process at the reef crest as observed in the experiments. In addition, the accurate simulation of the onset of the breaking process and the subsequent wave attenuation provided a good estimate of the wave setup on the reef flat.

Predicted evolution of the spectral shape agreed well with observations, including dissipation of energy around spectral peak by breaking, and the dominated infragravity motions trapped over reef flat and subsequently resonantly amplified at shoreline.

Only the bottom friction coefficient was calibrated for the best model-data match in one (arbitrary) test condition. This demonstrates the robustness of SWASH concerning its skillfulness to predict the wave transformation across fringing reefs for a wide range of different wave conditions. 


\section{REFERENCES}

Demirbilek, Z., O.G. Nwogu, and D.L. Ward. 2007. Laboratory study of wind effect on runup over fringing reefs, Report 1: data report, U.S. Army Engineer Research and Development Center, Vicksburg, Mississipi, Rep. No. ERDC/CHL-TR-07-4.

Filipot, J.-F., and K.F. Cheung. 2012. Spectral wave modeling in fringing reef environments, Coastal Engineering, 67, 67-79.

Massel, S.R., and M.R. Gourlay. 2000. On the modelling of wave breaking and set-up on coral reefs, Coastal Engineering, 39, 1-27.

Monismith, S. G. 2007. Hydrodynamics of coral reefs, Annual Review of Fluid Mechanics, 39, 35-55.

Nwogu, O., and Z. Demirbilek. 2010. Infragravity wave motions and runup over shallow fringing reefs, Journal of Waterway, Port, Coastal, and Ocean Engineering, 136, 295-305.

Peregrine, D.H. 1983. Breaking waves on beaches, Annual Review of Fluid Mechanics, 15, 149-178.

Rijnsdorp, D.P., P.B. Smit, and M. Zijlema. 2012. Non-hydrostatic modelling of infragravity waves using SWASH. Proceedings of $33^{\text {rd }}$ International Conference on Coastal Engineering, ASCE, this volume.

Roeber, V., and K.F. Cheung. 2012. Boussinesq-type model for energetic breaking waves in fringing reef environments, Coastal Engineering, 70, 1-20.

Sheremet, A., J.M. Kaihatu, S.-F. Su, E.R. Smith, and J.M. Smith. 2011. Modeling of nonlinear wave propagation over fringing reefs, Coastal Engineering, 58, 1125-1137.

Skotner, C., and C.J. Apelt. 1999. Application of a Boussinesq model for the computation of breaking waves. Part 2: Wave-induced setdown and setup on a submerged coral reef, Ocean Engineering, 26, 927-947.

Smit, P.B., M. Zijlema, and G.S. Stelling. 2012. Depth-induced wave breaking in a non-hydrostatic, near-shore wave model, Coastal Engineering, submitted.

Stelling, G.S., and S.P.A. Duinmeijer. 2003. A staggered conservative scheme for every Froude number in rapidly varied shallow water flows, International Journal of Numerical Methods in Fluids, 43, 1329-1354.

Stelling, G., and M. Zijlema. 2003. An accurate and efficient finite-difference algorithm for nonhydrostatic free-surface flow with application to wave propagation, International Journal of Numerical Methods in Fluids, 43, 1-23.

Wesseling, P. 2001. Principles of Computational Fluid Dynamics, Springer-Verlag, Berlin, 644 pp.

Zijlema, M., and G.S. Stelling. 2005. Further experiences with computing non-hydrostatic free-surface flows involving water waves, International Journal of Numerical Methods in Fluids, 48, 169-197.

Zijlema, M., and G.S. Stelling. 2008. Efficient computation of surf zone waves using the nonlinear shallow water equations with non-hydrostatic pressure, Coastal Engineering, 55, 780-790.

Zijlema, M., G. Stelling, and P. Smit. 2011. SWASH: An operational public domain code for simulating wave fields and rapidly varied flows in coastal waters, Coastal Engineering, 58, 9921012. 